\title{
Herbal acupuncture for type 2 diabetes: A meta-analysis
}

\author{
SEUNG-WOOK LEE ${ }^{1 *}$, MIN-HO NAM ${ }^{2 *}$ and BYUNG-CHEOL LEE ${ }^{1}$ \\ Departments of ${ }^{1}$ Clinical Korean Medicine and ${ }^{2}$ Science in Korean Medicine, Graduate School, \\ Kyung Hee University, Dongdaemun-Gu, Seoul 02447, Republic of Korea
}

Received August 28, 2015; Accepted January 26, 2017

DOI: 10.3892/etm.2017.4379

\begin{abstract}
Herbal acupuncture (HA) is a modern adjunctive technique in which natural herbs or biologic substances are injected into acupuncture points. The objective of this systematic review was to evaluate evidence of the effectiveness of HA for type 2 diabetes mellitus (T2DM). Three databases were searched. The included randomized controlled trials (RCTs) evaluated HA in controls and patients with T2DM and reported at least one of the following: Fasting blood glucose (FBG), postprandial (PP2hr) glucose and glycated hemoglobin (HbA1c). In a meta-analysis of seven RCTs ( $n=598$ patients), HA significantly reduced levels of FBG, PP2hr glucose and HbA1c $(\mathrm{P}<0.0001, \mathrm{P}=0.0005$ and $\mathrm{P}=0.004$, respectively). There was no significant effect of HA on total cholesterol, high-density lipoprotein (HDL) cholesterol, low-density lipoprotein (LDL) cholesterol, or triglyceride level. High degrees of heterogeneity were present for all analyses except HDL-cholesterol and LDL-cholesterol. HA might have a beneficial effect on FBG, PP2hr glucose and HbAlc levels in patients with T2DM; however, because of several limitations, the results are inconclusive. Therefore, additional rigorous RCTs are warranted to overcome the limitations of previous studies.
\end{abstract}

\section{Introduction}

Diabetes is a metabolic syndrome associated with hyperglycemia, obesity, insulin resistance, hypertension, complex dyslipidemia, atherosclerosis and endothelial dysfunction (1-3). The global prevalence of diabetes is increasing. This trend has been attributed to numerous factors, including rapid changes in eating habits, increasingly sedentary lifestyles and genetic

Correspondence to: Professor Byung-Cheol Lee, Department of Clinical Korean Medicine, Graduate School, Kyung Hee University, 26 Kyungheedae-ro, Dongdaemun-Gu, Seoul 02447, Republic of Korea

E-mail: hydrolee@korea.com

*Contributed equally

Key words: herbal acupuncture, pharmacopuncture, diabetes, fasting blood glucose, postprandial glucose, glycated hemoglobin factors that determine body fat distribution (4). Appropriate interventions to control and prevent hyperglycemia are central to the management of diabetes (5-9). While drugs, diet and physical activity are key to treatment strategies, there is a growing interest in complementary and alternative medicine for diabetes, not only among the general public, but also among health care providers, researchers and educators (10). Herbal remedies and acupuncture could prove to be effective alternative and adjunctive treatments for diabetes. Recent clinical trials using herbal medicine $(11,12)$ and acupuncture $(13,14)$ have reported possible therapeutic value in diabetes, although these results are unreliable due to small sample sizes and low-quality methodologies.

Herbal acupuncture (HA), also known as pharmacopuncture, acupuncture point injection, acupoint injection, or point injection therapy, is a modern adjunctive technique that utilizes the injection of natural herbs or biologic substances into acupuncture points to promote, maintain or restore health and to prevent disease $(15,16)$. To date, this approach has been applied to relieve the pain associated with diseases such as rheumatoid arthritis, osteoarthritis and musculoskeletal disease. Only a small number of studies have been conducted to evaluate pharmacopuncture applications in various diseases, including obesity and digestive diseases $(15,17)$. Thus, this systematic review summarizes the findings related to HA, focusing on its efficacy, safety and possible therapeutic value in the treatment of type 2 diabetes mellitus (T2DM).

\section{Materials and methods}

Search strategy. We performed a systematic literature search from January 2000 to February 2015. The following databases were included to identify relevant studies: MEDLINE through Pubmed, the Chinese National Knowledge Infrastructure (CNKI) and the Oriental Medicine Advanced Searching Integrated System (OASIS). The references in all located articles were searched manually for further relevant articles. Key terms were searched as follows: [(Diabetes OR diabetes mellitus) and (pharmacopuncture OR herbal acupuncture OR acupoint injection OR acupuncture point injection)]. CNKI and OASIS are databases from China and the Korea, respectively, so Chinese and Korean terms that corresponded to the original key words were also searched.

Study selection. The current review included all identified RCTs that assessed the efficacy of HA in diabetes treatment. 
The quality and language of studies was not regarded at this stage of study analysis. Duplicate papers that reported the same or similar results were excluded. In particular, the same terms in different languages caused a number of paper duplications. Conference abstracts with no subsequent publication and dissertations were disregarded.

Studies that dealt with diabetes patients were included, while studies focusing on type 1 diabetes or other diseases with diabetes were not included. Studies that tested pharmacopuncture as part of a more complex intervention were also included if a control group used the same complex interventions. However, studies with acupoint injection without herbal medicine were excluded; most of these studies used vitamins, scopolamine, loperamide, mecobalamin or metoclopramide.

In order to analyze efficacy, studies that included data on at least one of the following were included and assessed: Fasting blood glucose (FBG), postprandial (PP2hr) glucose or glycated hemoglobin (HbAlc). In the included studies, FBG, PP2hr glucose, HbAlc, total cholesterol, high-density lipoprotein (HDL) cholesterol, low-density lipoprotein (LDL) cholesterol and triglyceride (TG) levels were assessed.

Data extraction and quality assessment. Three reviewers independently selected relevant studies based on title and abstract. Full text versions of potentially eligible studies were retrieved and reviewed separately based on the aforementioned criteria. Data were extracted from original articles by the three reviewers. The results of each study were abstracted by a single reviewer and reassessed by the others. The Cochrane risk of bias assessment was used to evaluate methodological quality (18). Disagreements concerning the eligibility for inclusion were resolved through discussion.

Data synthesis. In order to summarize the effects of HA on each outcome, the risk estimates were abstracted. The standardized mean difference and $95 \%$ confidence interval (CI) were calculated using Review Manager version 5.3 (The Cochrane Collaboration, Oxford, UK). $\mathrm{P}<0.05$ was considered to indicate a statistically significant result. The variance of change was imputed using a correlation factor of 0.4. Data was then pooled across studies, statistical heterogeneity was evaluated using $\chi^{2}$ and Higgins $\mathrm{I}^{2}$ tests and $\mathrm{P}<0.10$ was considered significant. A random effects model was used if heterogeneity across studies was present.

\section{Results}

Description of studies. A total of 271 potentially relevant articles from the CNKI database were considered. After screening the abstracts and titles, 254 articles were excluded (Fig. 1). A detailed evaluation of the remaining 17 articles was performed by reading full texts, which determined that eight of the articles were duplicates. Full texts of two articles were not available. Consequently, seven RCTs met our inclusion criteria and were included in this review. The key data are summarized in Table I. All of the studies were performed and published in China, and reported positive effects of HA on T2DM.

Four studies used Western medical treatments such as gliclazide (19), metformin (20), glibenclamide (21) and insulin potentiation therapy (22) for both HA and control groups. One

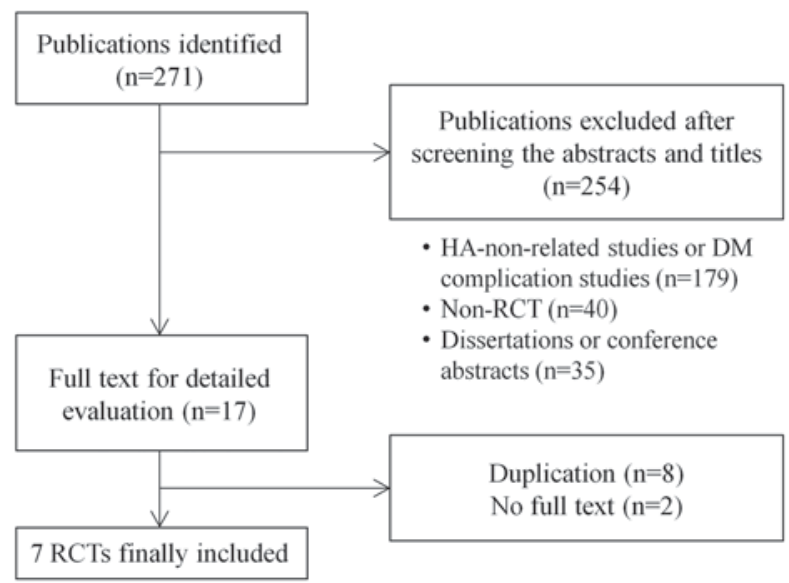

Figure 1. Flow chart of the study selection process. RCT, randomized controlled trial; HA, herbal acupuncture; DM, diabetes mellitus.

study (20) used oral administration of Yiqi Zishen decoction in addition to HA. These RCTs studied the effect of HA as an adjuvant therapy. While most of the studies evaluated HA effects compared to those of Western medical treatments, one study tested HA vs. Xiaoke Wan, a traditional Chinese medicine (TCM) decoction (23), and another tested HA vs. life-style interventions (24). Finally, one study tested the effect of HA treatment with various controls, such as saline injection into acupoints and muscle injection of herb extracts (21).

The seven included RCTs evaluated 628 (mean sample size, 90) patients with T2DM. A total of 300 patients received HA treatment for a range of 14 to 40 days (mean, 27 days). The outcome measures of these trials were FBG, PP2hr glucose, HbAlc, insulin resistance index, plasma insulin, total, HDL and LDL cholesterol, and TG. Baseline comparisons of major parameters related to diabetes were reported in all studies.

Herbal acupuncture treatments. Three types of herb extract were used for HA treatment in the reviewed RCTs: Astragalus propinquus (also known as Astragalus membranaceus), Salvia miltiorrhiza and a mixture of Angelica sinensis and vitamin B12. A. propinquus was used in four studies and $S$. miltiorrhiza was used in three studies. One study used these two herb extracts but alternated the herbs in the included patients. A mixture of A. sinensis and vitamin B12 was used in one study.

Six studies used multiple acupoints, whereas one study used a single acupoint (Table II) (21). There were 18 target acupoints, including 17 meridian points and one extra point. The most commonly used point was SP6, and the next most common was ST36. Among 18 acupoints, five were located on the lower extremities (ST36, SP6, SP9, SP10 and KI3), two on the upper extremities (LI4 and LI11), three on the abdomen (CV4, CV6 and CV12) and the remaining on the back (BL13, BL17, BL20, BL21, BL22, BL23, BL52 and Yishu). The majority of the acupoints were related to the gastrointestinal tract (ST36, SP6, SP9, SP10, LI4, LI11, CV4, CV6, CV12, BL20 and BL21) or kidneys (KI3, BL23 and BL52). All RCTs stated the rationale for acupuncture point selection from TCM theory.

Overall effects of herbal acupuncture. Seven RCTs (19-25) assessed the effects of HA on FBG, five RCTs on PP2hr 


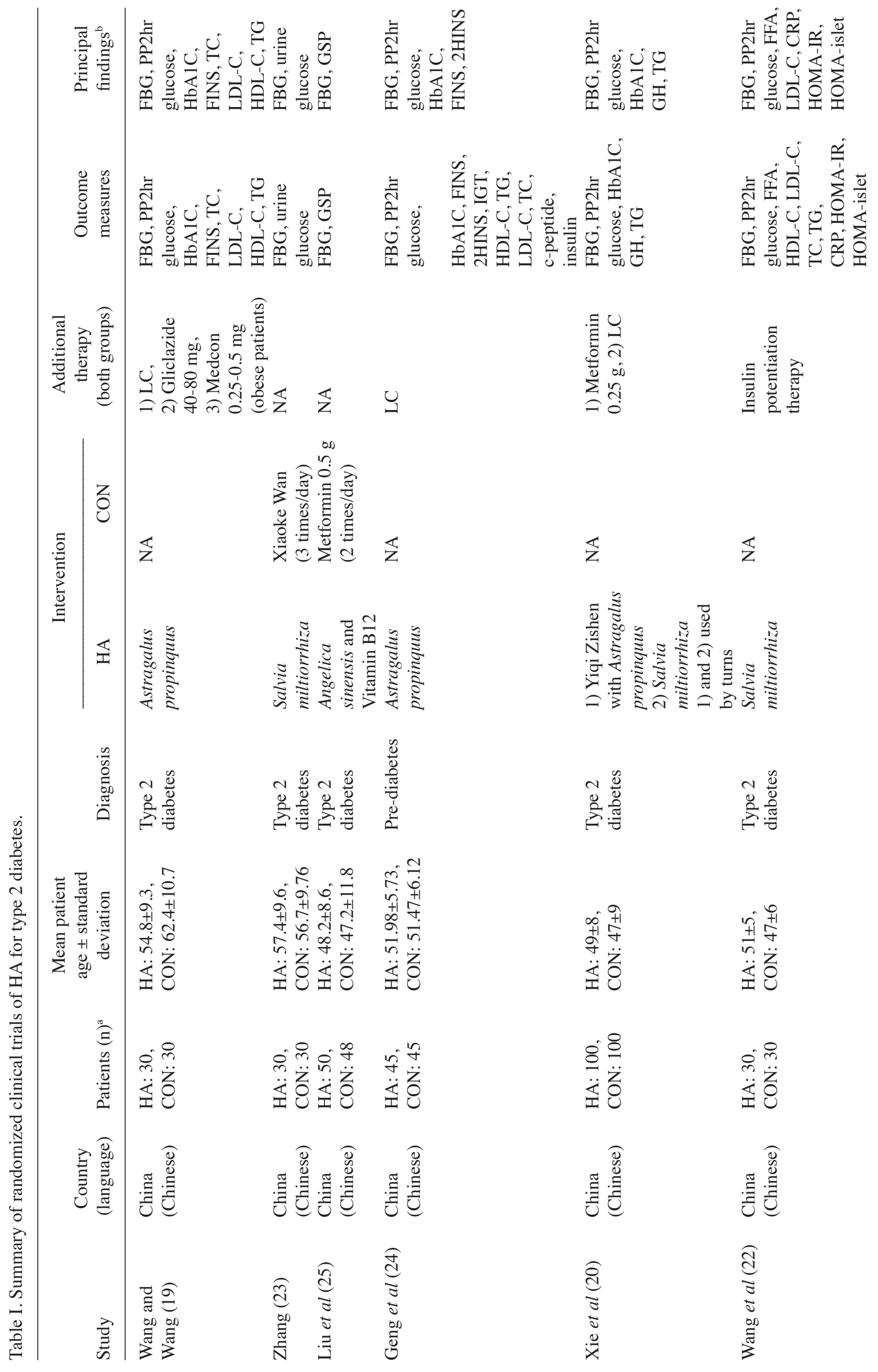


glucose, and four RCTs on HbAlc compared to controls. Upon meta-analysis, HA was associated with statistically significant reductions in levels of FBG (mean difference, $-1.16 \mathrm{mmol} / \mathrm{l}$; 95\% CI, -0.63 to $-1.69 \mathrm{mg} / \mathrm{dl}, \mathrm{P}<0.0001$; heterogeneity: $\chi^{2}=44.74, \mathrm{P}<0.0001, \mathrm{I}^{2}=87 \%$ ), PP2 $\mathrm{hr}$ glucose (mean difference, $-1.34 \mathrm{mmol} / \mathrm{l} ; 95 \% \mathrm{CI},-0.58$ to- $2.09 \mathrm{mg} / \mathrm{dl}, \mathrm{P}=0.0005$; heterogeneity: $\left.\chi^{2}=36.78, \mathrm{P}<0.0001, \mathrm{I}^{2}=89 \%\right)$ and HbA1c (mean difference, $-0.80 \%$; $95 \% \mathrm{CI},-0.25$ to- $1.35 \%, \mathrm{P}=0.004$; heterogeneity: $\chi^{2}=22.17, \mathrm{P}=0.0002, \mathrm{I}^{2}=85 \%$ ) compared with the control group (Fig. 2). However, there were no significant effects of HA on total cholesterol (mean difference, $-0.18 \mathrm{mmol} / 1 ; 95 \%$ CI, 0.09 to $-0.44 \mathrm{mmol} / 1$; heterogeneity: $\chi^{2}=12.06, \mathrm{P}=0.007$, $\mathrm{I}^{2}=75 \%$ ), HDL cholesterol (mean difference, $0.08 \mathrm{mmol} / \mathrm{l}$; 95\% CI, 0.17 to- $0.01 \mathrm{mmol} / \mathrm{l}$; heterogeneity: $\chi^{2}=0.33, \mathrm{P}=0.85$, $\mathrm{I}^{2}=0 \%$ ), LDL cholesterol (mean difference, $-0.11 \mathrm{mmol} / \mathrm{l}$; 95\% CI, 0.09 to $-0.31 \mathrm{mmol} / \mathrm{l}$; heterogeneity: $\chi^{2}=4.19, \mathrm{P}=0.12$, $\mathrm{I}^{2}=52 \%$ ) or TG (mean difference, $-0.25 \mathrm{mmol} / \mathrm{l} ; 95 \% \mathrm{CI}, 0.05$ to $-0.56 \mathrm{mmol} / \mathrm{l}$; heterogeneity: $\chi^{2}=12.33, \mathrm{P}=0.006, \mathrm{I}^{2}=76 \%$ ) compared with the control group (Fig. 3).

Subgroup analysis for outcomes of HA was performed based on the control types (sham treatment, conventional treatment or lifestyle intervention).

Herbal acupuncture vs. sham treatment. Four RCTs (19-22) evaluated the effectiveness of HA in comparison to sham treatment that was supplemental to anti-hyperglycemia therapy, such as gliclazide, glibenclamide, metformin or insulin. All RCTs indicated a favorable effect of HA compared to sham treatment with anti-hyperglycemia therapy in relation to FBG, $\mathrm{PP} 2 \mathrm{hr}$ glucose and HbAlc. The meta-analysis also revealed that HA showed a significant favorable effect on FBG (four studies, $\mathrm{n}=175$; mean difference, 1.48 ; $95 \% \mathrm{CI}, 0.63$ to 2.32; $\mathrm{P}=0.0006$; heterogeneity: $\chi^{2}=22.35, \mathrm{P}<0.0001, \mathrm{I}^{2}=87 \%$ ), PP $2 \mathrm{hr}$ glucose (four studies, $\mathrm{n}=175$; mean difference, $1.80 ; 95 \% \mathrm{CI}$, 0.72 to 2.87; $\mathrm{P}=0.001$; heterogeneity: $\chi^{2}=20.45, \mathrm{P}=0.0001$, $\mathrm{I}^{2}=85 \%$ ) and HbA1c (four studies, $\mathrm{n}=175$; mean difference, 1.12; $95 \%$ CI, 0.16 to $2.09 ; \mathrm{P}=0.02$; heterogeneity: $\chi^{2}=15.07$, $\left.\mathrm{P}=0.0005, \mathrm{I}^{2}=87 \%\right)$.

Three RCTs $(19,20,22)$ analyzed the effect of HA on total cholesterol and TG, demonstrating that that HA had superior effects compared with the control treatment. However, no statistical difference was found in the meta-analysis between the $\mathrm{HA}$ and sham groups (total cholesterol, $\mathrm{P}=0.26$; $\mathrm{TG}, \mathrm{P}=0.24$ ). Two RCTs also assessed the effect of HA on HDL-cholesterol and LDL-cholesterol, but no significant effects of HA were observed in the meta-analysis (HDL-cholesterol, $\mathrm{P}=0.56$; LDL-cholesterol, $\mathrm{P}=0.15$ ).

Herbal acupuncture vs. conventional treatment. Two RCTs $(23,25)$ assessed the effects of HA compared to conventional treatment, such as metformin or Xiaoke Wan (a herbal remedy). All RCTs showed a positive effect of HA on FBG compared to conventional treatment, and additional meta-analysis revealed that $\mathrm{HA}$ showed a significant favorable effect on FBG (two studies, $\mathrm{n}=80$; mean difference, 1.14; 95\% CI, 0.60 to 1.68; $\mathrm{P}<0.0001$; heterogeneity: $\chi^{2}=0.94, \mathrm{P}=0.33$, $\mathrm{I}^{2}=0 \%$.

Side effects. Three studies $(20,22,24)$ mentioned adverse events related to HA. Two studies $(20,24)$ reported no adverse events 
Table II. Herbal acupuncture intervention details.

\begin{tabular}{|c|c|c|}
\hline Study & Acupuncture points & Injection methods \\
\hline $\begin{array}{l}\text { Wang and } \\
\text { Wang (19) }\end{array}$ & SP6, BL20, Yishu & Bilateral, $1.5 \mathrm{ml}$, q.d. for 20 days \\
\hline Zhang (23) & $\begin{array}{l}\text { ST36, KI3, SP6, SP10, LI4, BL20, } \\
\text { BL21, BL22, BL23, BL13, Yishu }\end{array}$ & $\begin{array}{l}\text { Bilateral, } 1 \mathrm{ml}, 2 \text { sets of q.o.d. for } \\
20 \text { days with } 1 \text {-week interval }\end{array}$ \\
\hline Liu et al (25) & $\begin{array}{l}\text { BL20, BL17, BL23, BL52, } \\
\text { ST36, SP6, KI3, Yishu }\end{array}$ & $\begin{array}{l}\text { Unilateral in turn, } 0.5 \mathrm{ml}, 2 \text { sets of } \\
\text { q.o.d. for } 20 \text { days with } 5 \text {-day interval }\end{array}$ \\
\hline Geng et al (24) & $\begin{array}{l}\text { 1) CV12, ST36, Yishu } \\
\text { 2) SP6, BL23, CV4 }\end{array}$ & $\begin{array}{l}\text { Alternating applications of 1) or 2) were } \\
\text { applied. } 3 \text { sets of q.d. for } 10 \text { days } \\
\text { with } 5 \text {-day intervals }\end{array}$ \\
\hline Xie et al (20) & ST36, SP6, LI11, BL23, CV6 & $0.5 \mathrm{ml}, \mathrm{q} . \mathrm{d}$. for 4 weeks \\
\hline Wang et al (22) & $\begin{array}{l}\text { 1) left SP9, right ST36 } \\
\text { 2) right SP9, left ST36 }\end{array}$ & $\begin{array}{l}\text { 1) or 2) was applied in turns. } \\
1 \mathrm{ml} \text {, q.d. for } 2 \text { weeks. }\end{array}$ \\
\hline Li et al (21) & SP6 & Bilateral, $2 \mathrm{ml}$, every 3 days for 18 days \\
\hline
\end{tabular}

q.d., every day; q.o.d, every other day.

\begin{tabular}{|c|c|c|c|c|c|c|}
\hline \multicolumn{7}{|c|}{ A. FBG (mmol/I) } \\
\hline Study name & Control & Herbal Acupuncture & Weight & Mean Difference, $95 \% \mathrm{Cl}$ & \multicolumn{2}{|c|}{ Mean Difference, $95 \% \mathrm{Cl}$} \\
\hline \multicolumn{7}{|c|}{ 1. Herbal Acupuncture vs Sham treatment } \\
\hline Li et al, 2000 & 15 & 15 & 10.6 & $2.22[1.17,3.27]$ & & \\
\hline Wang et al, 2002 & 30 & 30 & 13.0 & $2.25[1.46,3.04]$ & & - \\
\hline Wang et al, 2012 & 30 & 30 & 16.5 & $0.45[0.03,0.87]$ & & 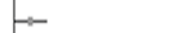 \\
\hline Xie et al, 2012 & 100 & 100 & 16.0 & $1.30[0.83,1.77]$ & & \\
\hline Subtotal & & & & $1.48[0.63,2.32]$ & & \\
\hline \multicolumn{7}{|c|}{ 2. Herbal Acupuncture vs Conventional treatment } \\
\hline Zhang et al, 2002 & 30 & 30 & 14.1 & $1.34[0.66,2.02]$ & & \\
\hline Liu et al, 2008 & 48 & 50 & 11.9 & $0.78[-0.13,1.69]$ & & \\
\hline Subtotal & & & & $1.14[0.60,1.68]$ & & \\
\hline \multicolumn{7}{|c|}{ 3. Herbal Acupuncture vs Lifestyle change } \\
\hline Geng et al, 2014 & 45 & 45 & 18.0 & $0.39[0.21,0.57]$ & & + \\
\hline Subtotal & & & & $0.39[0.21,0.57]$ & & $\Delta$ \\
\hline Total & & & & $1.16[0.63,1.69]$ & & \\
\hline $\begin{array}{l}\text { Heterogeneity: Ta } \\
\text { Test for overall ef }\end{array}$ & $\begin{array}{l}\text { au }^{2}=0.39 \\
\text { ffects: } Z=\end{array}$ & $\begin{array}{l}\mathrm{Chi}^{2}=44.74, \mathrm{df}=6(\mathrm{P} \\
4.31(\mathrm{P}<0.0001)\end{array}$ & $0.00001)$ & $2=87 \%$ & ${ }^{-4}{ }_{\text {Favors Control }}^{-2}$ & $\begin{array}{cc}3 & 4 \\
\text { Favors treatment }\end{array}$ \\
\hline \multicolumn{7}{|c|}{ B. PP2hrs glucose (mmol/I) } \\
\hline \multirow[b]{2}{*}{ Study name } & \multirow{2}{*}{$\frac{\mathrm{S}}{\text { Control }}$} & ample size & & & & \\
\hline & & Herbal Acupuncture & Weight & Mean Difference, $95 \%$ & \multicolumn{2}{|c|}{ Mean Difference, $95 \% \mathrm{Cl}$} \\
\hline \multicolumn{7}{|c|}{ 1. Herbal Acupuncture vs Sham treatment } \\
\hline Li et al, 2000 & 15 & 15 & 9.3 & $4.52[2.52,6.52]$ & & \\
\hline Wang et al, 2002 & 30 & 30 & 17.3 & $2.42[1.35,3.49]$ & & \\
\hline Wang et al, 2012 & 30 & 30 & 23.6 & $0.60[0.07,1.13]$ & & - \\
\hline Xie et al, 2012 & 100 & 100 & 23.3 & $1.10[0.54,1.66]$ & & \\
\hline Subtotal & & & & $1.80[0.72,2.87]$ & & \\
\hline \multicolumn{7}{|c|}{ 2. Herbal Acupuncture vs Lifestyle change } \\
\hline Geng et al, 2002 & 45 & 45 & 26.5 & $0.37[0.28,0.46]$ & & 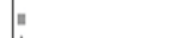 \\
\hline Subtotal & & & & $0.37[0.28,0.46]$ & & 1 \\
\hline Total & & & & $1.34[0.58,2.09]$ & & \\
\hline \multicolumn{5}{|c|}{$\begin{array}{l}\text { Heterogeneity: } \mathrm{Tau}^{2}=0.56 ; \mathrm{Chi}^{2}=36.78, \mathrm{df}=4(\mathrm{P}<0.00001) ; \mathrm{I}^{2}=89 \% \\
\text { Test for overall effects: } Z=3.46(P=0.0005)\end{array}$} & \multirow{2}{*}{\multicolumn{2}{|c|}{ Mean Difference, $95 \% \mathrm{Cl}$}} \\
\hline \multicolumn{7}{|l|}{ C. HbA1c (\%) } \\
\hline Study name & Control & Herbal Acupuncture & Weight & Mean Difference, $95 \%$ & & \\
\hline \multicolumn{7}{|c|}{ 1. Herbal Acupuncture vs Sham treatment } \\
\hline Li et al, 2000 & 15 & 15 & 16.6 & $2.30[1.35,3.25]$ & & - \\
\hline Wang et al, 2002 & 30 & 30 & 22.9 & $0.97[0.34,1.60]$ & & 一 \\
\hline Xie et al, 2012 & 100 & 100 & 29.0 & $0.37[0.02,0.72]$ & & \\
\hline Subtotal & & & & $1.12[0.16,2.09]$ & & \\
\hline \multicolumn{7}{|c|}{ 2. Herbal Acupuncture vs Lifestyle change } \\
\hline Geng et all, 2014 & 45 & 45 & 31.5 & $0.28[0.09,0.47]$ & & 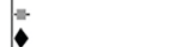 \\
\hline Subtotal & & & & $0.28[0.09,0.47]$ & & \\
\hline Total & & & & $0.80[0.25,1.35]$ & & \\
\hline $\begin{array}{l}\text { Heterogeneity: Ta } \\
\text { Test for overall ef }\end{array}$ & $\begin{array}{l}a u^{2}=0.24 \\
\text { ffects: } Z=2\end{array}$ & $\begin{array}{l}\mathrm{Chi}^{2}=20.17, \mathrm{df}=3(\mathrm{P} \\
2.85(\mathrm{P}=0.004)\end{array}$ & $.0002) ; 1$ & $=85 \%$ & $\begin{array}{l}1 \\
-4 \\
\text { Favors Control }\end{array}$ & Favors tr \\
\hline
\end{tabular}

Figure 2. Forest plot depicting the meta-analysis results of randomized controlled trials that evaluated the effects of herbal acupuncture on (A) FBG, (B) PP2hrs glucose and (C) HbA1c. FBG, fasting blood glucose; PP2hrs, Postprandial 2 h; HbA1C, glycated hemoglobin; CI, confidence interval. 
A. Total Cholesterol (mmol/l)

Sample size

Study name Control Herbal Acupuncture Weight Mean Difference, $95 \% \mathrm{Cl}$ Mean Difference, $95 \% \mathrm{Cl}$

1. Herbal Acupuncture vs Sham treatment

Wang et al, $2002 \quad 30 \quad 30 \quad 13.3 \quad 0.48[-0.11,1.07]$

Wang et al, $2012 \quad 30 \quad 30 \quad 29.5 \quad-0.10[-0.33,0.13]$

Xie et al, $2012 \quad 100 \quad 100 \quad 21.8 \quad 0.60[0.23,0.97]$

Subtotal $\quad 0.30[-0.22,0.81]$

2. Herbal Acupuncture vs Lifestyle change

Geng et al, $2014 \quad 45 \quad 45 \quad 35.5 \quad 0.03[-0.08,0.14]$

Subtotal $\quad 0.03[-0.08,0.14]$

Total

Heterogeneity: $\mathrm{Tau}^{2}=0.05 ; \mathrm{Chi}^{2}=12.06, \mathrm{df}=3(\mathrm{P}=0.007) ; \mathrm{I}^{2}=75 \%$

Test for overall effects: $Z=1.28(P=0.20)$

$0.18[-0.09,0.44]$

B. HDL Cholesterol (mmol/I)

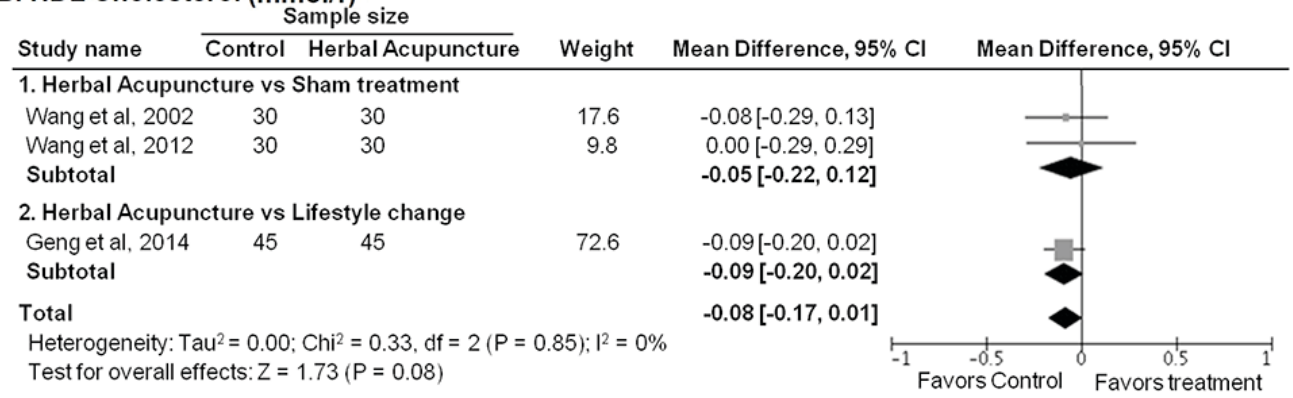

C. LDL Cholesterol (mmol/I)

\begin{tabular}{|c|c|c|c|c|c|}
\hline \multirow[b]{2}{*}{ Study name } & \multicolumn{2}{|c|}{ Sample size } & \multirow[b]{2}{*}{ Weight } & \multirow[b]{2}{*}{ Mean Difference, $95 \% \mathrm{Cl}$} & \multirow[b]{2}{*}{ Mean Difference, $95 \% \mathrm{C}$} \\
\hline & Control & Herbal Acupuncture & & & \\
\hline \multicolumn{5}{|c|}{ 1. Herbal Acupuncture vs Sham treatment } & \\
\hline Wang et al, 2002 & 30 & 30 & 27.6 & $0.04[-0.24,0.32]$ & 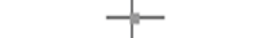 \\
\hline Wang et al, 2012 & 30 & 30 & 34.5 & $0.30[0.07,0.53]$ & \\
\hline Subtotal & & & & $0.18[-0.07,0.44]$ & \\
\hline \multicolumn{5}{|c|}{ 2. Herbal Acupuncture vs Lifestyle change } & \\
\hline Geng et al, 2014 & 45 & 45 & 38.0 & $-0.01[-0.22,0.20]$ & \\
\hline Subtotal & & & & $-0.01[-0.22,0.20]$ & \\
\hline \multirow{2}{*}{\multicolumn{5}{|c|}{$\begin{array}{l}\text { Total } \\
\text { Heterogeneity: } \mathrm{Tau}^{2}=0.02 ; \mathrm{Chi}^{2}=4.19 . \mathrm{df}=2(\mathrm{P}=0.12): \mathrm{I}^{2}=52 \%\end{array}$}} & \\
\hline & & $\begin{array}{l}C h i^{2}=4.19, d f=2(P=0 \\
1.09(P=0.27)\end{array}$ & 12); $\left.\right|^{2}=52 \%$ & -2 & trol $\mathrm{Fav}$ \\
\hline
\end{tabular}

D. Triglyceride (mmol//)

\begin{tabular}{|c|c|c|c|c|c|}
\hline Study name & Control & Herbal Acupuncture & Weight & Mean Difference, $95 \% \mathrm{Cl}$ & Mean Difference, $95 \% \mathrm{C}$ \\
\hline \multicolumn{6}{|c|}{ 1. Herbal Acupuncture vs Sham treatment } \\
\hline Wang et al, 2002 & 30 & 30 & 17.0 & $0.46[-0.05,0.97]$ & \\
\hline Wang et al, 2012 & 30 & 30 & 29.7 & $-0.10[-0.33,0.13]$ & $\longrightarrow$ \\
\hline Xie et al, 2012 & 100 & 100 & 20.9 & $0.70[0.27,1.13]$ & \\
\hline \multicolumn{5}{|c|}{ 2. Herbal Acupuncture vs Lifestyle change } & \\
\hline Geng et al, 2014 & 45 & 45 & 31.5 & $0.17[-0.22,0.36]$ & \\
\hline Subtotal & & & & $0.17[-0.22,0.36]$ & \\
\hline Total & & & & $0.25[-0.05,0.56]$ & \\
\hline
\end{tabular}

Figure 3. Forest plot depicting the meta-analysis results of randomized controlled trials evaluating the effects of herbal acupuncture on (A) total cholesterol, (B) HDL cholesterol, (C) LDL cholesterol and (D) triglyceride. HDL, high density lipoprotein; LDL, low density lipoprotein; CI, confidence interval.

in a total of 145 cases; while one study (22) reported two cases of mild hypoglycemia out of a total of 30 cases. None of the studies reported any serious adverse events resulting from HA treatment.

\section{Discussion}

To our knowledge, this is the first systematic review and meta-analysis of RCTs that examines the effectiveness of HA for managing patients with T2DM. The results from meta-analysis indicated that HA may have a beneficial effect on FBG, PP2hr glucose and HbA1c levels in patients with
T2DM, and suggest that HA may have clinical applications in the management of T2DM.

Understanding the respective efficacies of herbal remedies and acupuncture stimulation is necessary to understand the overall efficacy of HA because HA is a combination of these two approaches. HA therapy for improving conditions of patients with T2DM primarily used A. propinquus, $S$. miltiorrhiza and A. sinensis. A. propinquus is the most commonly used herb in anti-diabetic TCM decoctions and is known to contain rich anti-diabetic compounds, such as polysaccharides, saponins and flavonoids $(26,27)$. It has been reported to reduce complications of diabetes such as increased 
urinary albumin excretion, plasma creatinine and blood urea nitrogen levels (28), as well as diabetic nephropathy $(29,30)$. S. miltiorrhiza has also shown a significant anti-diabetic effect in animal studies; for instance, it decreases FBG and increases the insulin sensitivity index $(31,32)$. It is effective for preventing and treating diabetic complications such as myocardial malfunction $(33,34)$ and diabetic retinopathy $(35)$. A. sinensis is also one of the most frequently used herbs for diabetes in China (36) due to evidence of its anti-diabetic properties (37). These previous findings align with the results of studies in the current review, which showed improvement in insulin sensitivity and microvascular diseases in animal and human studies following HA treatment.

In the clinical studies analyzed in this review, acupoints SP6, ST36, BL23, Yishu, BL20 and KI3 were selected for use in multiple studies. Classical (manual) acupuncture and electroacupuncture at these acupoints have previously been reported to be effective for improving diabetic conditions. For example, Wang et al reported that manual acupuncture at multiple acupoints including SP6, BL23, BL20, Yishu and KI3 positively regulates glucose and lipid metabolism in T2DM patients (38). Additionally, electroacupuncture at ST36, BL20, BL23 and SP9 has been reported to reduce HbA1c and PBG2 h levels in impaired glucose tolerance patients (39). Similarly, Peplow and Baxter demonstrated that electroacupuncture at ST36 is beneficial for lowering blood glucose in T2DM rats (40). Altogether, these results suggest that the therapeutic effect of HA therapy on T2DM is attributable to a combination of anti-diabetic herbs and anti-diabetic acupoint stimulation.

There were several limitations to the current review. First, the intervention methods used in the studies were not identical, although the acupoints SP6 (six studies), ST36 (five studies), BL23 (four studies) and Yishu (four studies) were typically used, and A. propinquus (four studies) and S. miltiorrhiza (three studies) were typically used as herbal material. Second, three different types of control group were used; however, HA showed positive effects regardless of the control group type. Third, although all of these studies were considered to have relatively homogenous T2DM patients, statistical pooling was difficult due to a lack of sufficient raw data. Another possible source of bias is the fact that the included trials were conducted in China. Fourth, the total number of trials included in our review and meta-analysis, and the total sample size, were too small to allow for definitive conclusions.

Nevertheless, the review demonstrated a comprehensive search strategy, included RCT designs only, and used suggested methods for systematic reviews of interventions for T2DM. Future studies of HA in T2DM should be of adequate sample size based on power calculations; provide sufficient details on selection, dose, frequency, duration of therapeutic herbal injection and acupoints; include adequate comparative control groups; and minimize other internal and external biases.

Based on the currently available literature, HA seems to have a beneficial effect on FBG, PP2hr glucose and HbA1c levels in patients with T2DM. However, HA does not have a statistically significant effect on lipid profiles. Caution should be exercised in applying the results of this analysis to patient care due to the limited quantity and quality of evidence regarding $\mathrm{HA}$ as a treatment for T2DM.

\section{Acknowledgements}

This study was supported by the Traditional Korean Medicine Research and Development program funded by the Ministry of Health and Welfare through the Korea Health Industry Development Institute (grant no. HI13C0700).

\section{References}

1. Razi MT, Saadullah M, Murtaza G and Hassan W: Determination of the anti-diabetic effect of methanolic extract of Sphaeranthus indicus L. on alloxan induced diabetic rabbits. Lat Am J Pharm 30: 378-382, 2011.

2. Johansen Taber KA and Dickinson BD: Genomic-based tools for the risk assessment, management and prevention of type 2 diabetes. Appl Clin Genet 8: 1-8, 2015.

3. American Diabetes Association: Diagnosis and classification of diabetes mellitus. Diabetes Care 36 (Suppl 1): S67-S74, 2013

4. Seidell JC: Obesity, insulin resistance and diabetes - a worldwide epidemic. Br J Nutr 83: S5-S8, 2000.

5. Brunisholz KD, Briot P, Hamilton S, Joy EA, Lomax M, Barton N, Cunningham R, Savitz LA and Cannon W: Diabetes self-management education improves quality of care and clinical outcomes determined by a diabetes bundle measure. J Multidiscip Healthc 7: 533-542, 2014.

6. Duncan I, Birkmeyer C, Coughlin S, Li QE, Sherr D and Boren S: Assessing the value of diabetes education. Diabetes Educ 35: 752-760, 2009.

7. Powers MA, Bardsley J, Cypress M, Duker P, Funnell MM, Hess Fischl A, Maryniuk MD, Siminerio L and Vivian E: Diabetes self-management education and support in type 2 diabetes: A joint position statement of the American diabetes association, the American association of diabetes educators and the academy of nutrition and dietetics. Diabetes Care 38: 1372-1382, 2015

8. Akkati S, Sam KG and Tungha G: Emergence of promising therapies in diabetes mellitus. J Clin Pharmacol 51: 796-804, 2011.

9. Resnick HE, Foster GL, Bardsley J and Ratner RE: Achievement of american diabetes association clinical practice recommendations among U.S. adults with diabetes, 1999-2002: The national health and nutrition examination survey. Diabetes Care 29: 531-537, 2006

10. Bloomgarden ZT: American diabetes association 60th scientific sessions, 2000: Nutrition, lipids, and alternative medicine. Diabetes Care 23: 1847-1851, 2000.

11. Lin YJ, Ho TJ, Yeh YC, Cheng CF, Shiao YT, Wang CB, Chien WK, Chen JH, Liu X, Tsang H, et al: Chinese Herbal medicine treatment improves the overall survival rate of individuals with hypertension among type 2 diabetes patients and modulates in vitro smooth muscle cell contractility. PLoS One 10: e145109, 2015.

12. Hu Y, Zhou X, Liu P, Wang B, Duan DM and Guo DH: A comparison study of metformin only therapy and metformin combined with Chinese medicine jianyutangkang therapy in patients with type 2 diabetes: A randomized placebo-controlled double-blind study. Complement Ther Med 24: 13-18, 2016.

13. Firouzjaei A, Li GC, Wang N, Liu WX and Zhu BM: Comparative evaluation of the therapeutic effect of metformin monotherapy with metformin and acupuncture combined therapy on weight loss and insulin sensitivity in diabetic patients. Nutr Diabetes 6: e209, 2016.

14. Zhiyuan W, Ming Y, Jie J, Yi W, Tiansheng H, Mingfen L, Zhijie H, Zhenzhen G and Pang LM: Effect of transcutaneous electrical nerve stimulation at acupoints on patients with type 2 diabetes mellitus: A randomized controlled trial. J Tradit Chin Med 35: 134-140, 2015.

15. Lim C, Park S, Sun S and Lee K: Research on Korean pharmacopuncture in South Korea since 2007. J Pharmacopuncture 17: 15-21, 2014.

16. Cha E, Lee J, Lee S, Park M and Kim S: Single-dose toxicity of shinyanghur herbal acupuncture. J Pharmacopuncture 18: 67-75, 2015.

17. Kim EJ, Jang MK, Yoon EH, Jung CY, Nam DW, Lee SD and Kim KS: Efficacy of pharmacopuncture using root bark of Ulmus davidiana Planch in patients with knee osteoarthritis: A double-blind randomized controlled trial. J Acupunct Meridian Stud 3: 16-23, 2010. 
18. Julian PT and Higgins SG: Cochrane handbook for systematic reviews of interventions. The Cochrane Collaboration, 2011.

19. Wang CY and Wang WY: Clinical observation on thirty cases of type 2 diabetes treated by acupoint injection of astragalus injection. Zhongguo Zhong Yi Yao Ke Ji 9: 172-173, 2002. (In Chinese).

20. Xie XND, G. N, Yang YQ, et al: The curing effect of YIQI ZISHEN prescription combined acupoint injection therapy on kidney yin deficiency syndrome of type 2 diabetes. Modern Hospital 12: 38-39, 2012.

21. Li LX, Chen K and Zeng XL: Effect of Acupoint-injection on glucometabolism in patients of type 11 diabetes. Zhongguo Zhen Jiu 8: 493-495, 2000 (In Chinese).

22. Wang KC, Ren YJ, Chen JY, Xiang YL, Hong LY and Yong HL: Clinical effect of point injection with danshen injection in diagnosed patients with type 2 diabetes mellitus in intensive insulin therapy. Tianjin Journal of Traditional Chinese Medicine 29: 149-152, 2012.

23. Zhang XL: Applying acupoint-injection to treating 30 cases of 2 type of mellitus diabetes. Hunan Guiding Journal of TCMP 8: $130,2002$.

24. Geng Y, Duan A, Yang Y, et al: Comparison of the effects of pre-diabetes intervention and acupoint-injection intervention. Tang Niao Bing Xin Shi Jie 7: 5-7, 2014 (In Chinese).

25. Liu XH, Song YP and Zhai DH: Clinical observation on 50 cases of type 2 diabetes treated by acupoint injection. Jiang Su Zhong Yi Yao 40: 78, 2008 (In Chinese).

26. Zhang K, Pugliese M, Pugliese A and Passantino A: Biological active ingredients of traditional Chinese herb Astragalus membranaceus on treatment of diabetes: A systematic review. Mini Rev Med Chem 15: 315-329, 2015.

27. Agyemang K, Han L, Liu E, Zhang Y, Wang T and Gao X: Recent advances in Astragalus membranaceus anti-diabetic research: Pharmacological effects of its phytochemical constituents. Evid Based Complement Alternat Med 2013: 654643, 2013.

28. Wang ZS, Xiong F, Xie XH, Chen D, Pan JH and Cheng L: Astragaloside IV attenuates proteinuria in streptozotocin-induced diabetic nephropathy via the inhibition of endoplasmic reticulum stress. BMC Nephrol 16: 44, 2015.

29. Kim J, Moon E and Kwon S: Effect of Astragalus membranaceus extract on diabetic nephropathy. Endocrinol Diabetes Metab Case Rep 2014: 140063, 2014.

30. Li M, Wang W, Xue J, Gu Y and Lin S: Meta-analysis of the clinical value of Astragalus membranaceus in diabetic nephropathy. J Ethnopharmacol 133: 412-419, 2011.
31. Huang M, Wang P, Xu S, Xu W, Xu W, Chu K and Lu J: Biological activities of salvianolic acid B from Salvia miltiorrhiza on type 2 diabetes induced by high-fat diet and streptozotocin. Pharm Biol 53: 1058-1065, 2015.

32. Raoufi S, Baluchnejadmojarad T, Roghani M, Ghazanfari T, Khojasteh F and Mansouri M: Antidiabetic potential of salvianolic acid B in multiple low-dose streptozotocin-induced diabetes. Pharm Biol 53: 1803-1809, 2015.

33. Yu J, Fei J, Azad J, Gong M, Lan Y and Chen G: Myocardial protection by Salvia miltiorrhiza injection in streptozotocin-induced diabetic rats through attenuation of expression of thrombospondin- 1 and transforming growth factor- $\beta 1$. J Int Med Res 40: 1016-1024, 2012.

34. Qian S, Wang S, Fan P, Huo D, Dai L and Qian Q: Effect of Salvia miltiorrhiza hydrophilic extract on the endothelial biomarkers in diabetic patients with chronic artery disease. Phytother Res 26: 1575-1578, 2012.

35. Lian F, Wu L, Tian J, Jin M, Zhou S, Zhao M, Wei L, Zheng Y, Wang Y, Zhang M, et al: The effectiveness and safety of a danshen-containing Chinese herbal medicine for diabetic retinopathy: A randomized, double-blind, placebo-controlled multicenter clinical trial. J Ethnopharmacol 164: 71-77, 2015.

36. Zhi XY: Traditional Chinese medicine diagnosis and treatment of type 2 diabetes in Tianjin urban population. Zhong Xi Yi Jie He Xue Bao 7: 823-826, 2009 (In Chinese).

37. Wang K, Cao P, Shui W, Yang Q, Tang Z and Zhang Y: Angelica sinensis polysaccharide regulates glucose and lipid metabolism disorder in prediabetic and streptozotocin-induced diabetic mice through the elevation of glycogen levels and reduction of inflammatory factors. Food Funct 6: 902-909, 2015.

38. Wang Y, Liu ZC and Xu B: Efficacy analysis on type 2 diabetes mellitus treated with acupuncture in females. Zhongguo Zhen Jiu 34: 21-24, 2014 (In Chinese).

39. Meng H, Hao JD, Wang HC, Zhao JY, Zhao CL and Zhai X: Effects of different frequencies of electroacupuncture on blood glucose level in impaired glucose tolerance patients. Zhen Ci Yan Jiu 36: 220-223, 2011 (In Chinese).

40. Peplow PV and Baxter GD: Electroacupuncture for control of blood glucose in diabetes: Literature review. J Acupunct Meridian Stud 5: 1-10, 2012. 